\title{
Inter-network regions of the Sun at millimetre wavelengths
}

\author{
S. Wedemeyer-Böhm ${ }^{1,2}$, H. G. Ludwig ${ }^{3}$, M. Steffen ${ }^{4}$, J. Leenaarts ${ }^{5}$, and B. Freytag ${ }^{6}$ \\ 1 Institute of Theoretical Astrophysics, University of Oslo, PO Box 1029 Blindern, 0315 Oslo, Norway \\ e-mail: sven.wedemeyer@astro.uio.no \\ 2 Kiepenheuer-Institut für Sonnenphysik, Schöneckstraße 6, 79104 Freiburg, Germany \\ 3 Observatoire de Paris-Meudon, CIFIST/GEPI, 92195 Meudon Cedex, France \\ 4 Astrophysikalisches Institut Potsdam, An der Sternwarte 16, 14482 Potsdam, Germany \\ 5 Sterrekundig Instituut, Utrecht University, Postbus 80 000, 3508 TA Utrecht, The Netherlands \\ ${ }^{6}$ Centre de Recherche Astronomique de Lyon - École Normale Supérieure, 46 Allée d'Italie, 69364 Lyon Cedex 07, France
}

Received 2 April 2007/ Accepted 15 May 2007

\begin{abstract}
Aims. The continuum intensity at wavelengths around $1 \mathrm{~mm}$ provides an excellent way to probe the solar chromosphere and thus valuable input for the ongoing controversy on the thermal structure and the dynamics of this layer.

The synthetic continuum intensity maps for near-millimetre wavelengths presented here demonstrate the potential of future observations of the small-scale structure and dynamics of internetwork regions on the Sun.

Methods. The synthetic intensity/brightness temperature maps are calculated on basis of three-dimensional radiation (magneto-)hydrodynamic (MHD) simulations. The assumption of local thermodynamic equilibrium (LTE) is valid for the source function. The electron densities are also treated in LTE for most maps but also in non-LTE for a representative model snapshot. Quantities like intensity contrast, intensity contribution functions, spatial and temporal scales are analysed in dependence on wavelength and heliocentric angle.

Results. While the millimetre continuum at $0.3 \mathrm{~mm}$ originates mainly from the upper photosphere, the longer wavelengths considered here map the low and middle chromosphere. The effective formation height increases generally with wavelength and also from disk-centre towards the solar limb. The average intensity contribution functions are usually rather broad and in some cases they are even double-peaked as there are contributions from hot shock waves and cool post-shock regions in the model chromosphere. The resulting shock-induced thermal structure translates to filamentary brightenings and fainter regions in between. Taking into account the deviations from ionisation equilibrium for hydrogen gives a less strong variation of the electron density and with it of the optical depth. The result is a narrower formation height range although the intensity maps still are characterised by a highly complex pattern. The average brightness temperature increases with wavelength and towards the limb although the wavelength-dependence is reversed for the MHD model and the NLTE brightness temperature maps. The relative contrast depends on wavelength in the same way as the average intensity but decreases towards the limb. The dependence of the brightness temperature distribution on wavelength and disk-position can be explained with the differences in formation height and the variation of temperature fluctuations with height in the model atmospheres. The related spatial and temporal scales of the chromospheric pattern should be accessible by future instruments. Conclusions. Future high-resolution millimetre arrays, such as the Atacama Large Millimeter Array (ALMA), will be capable of directly mapping the thermal structure of the solar chromosphere. Simultaneous observations at different wavelengths could be exploited for a tomography of the chromosphere, mapping its three-dimensional structure, and also for tracking shock waves. The new generation of millimetre arrays will be thus of great value for understanding the dynamics and structure of the solar atmosphere.
\end{abstract}

Key words. Sun: chromosphere - Sun: radio radiation - submillimeter - hydrodynamics - radiative transfer

\section{Introduction}

Many details concerning the small-scale structure of the solar chromosphere remain an open issue despite the large progress during the last decades on the observational but also on the modelling side. Recent works suggest that the solar chromosphere within internetwork regions is a highly inhomogeneous and dynamic phemenon demanding for high spatial and temporal resolution on both sides (e.g., Al et al. 2002; Krijger et al. 2001; Ayres 2002; Wöger et al. 2006; Tritschler et al. 2007; Vecchio et al. 2007, and references therein) On the numerical side, the increasing computational power but also sophisticated new methods now allow for the necessary resolution. On the other hand, advanced instruments enable new highly-resolved observations of hitherto not achieved quality (e.g., with SOT onboard the
Hinode satellite, see, e.g., Tsuneta 2006). For a detailed comparison of observed images and three-dimensional simulations, synthetic intensity maps need to be calculated. Unfortunately, the chromospheric diagnostics so far available, such as the calcium resonance lines, require the treatment of deviations from local thermodynamic equilibrium (LTE). A proper treatment demands for large computational resources and sophisticated methods, rendering such calculations hardly feasible for 3D models. And even in case of successful calculations, the complex translation of temperature into emergent intensity complicates the interpretation and hampers deriving the thermal structure from observations.

An exception, in contrast to most other diagnostics, is the radio continuum at millimetre and sub-millimetre wavelengths. As its source function can be treated in LTE, it can be 
synthesised easily and hence offers a convenient way to compare observations and numerical models. Loukitcheva et al. (2004, 2006) took advantage of this fact and compared a large collection of observations in the millimetre and sub-millimetre range with synthetic brightness temperatures calculated from models by Fontenla et al. (1993, hereafter FAL) and Carlsson \& Stein (1995, hereafter CS). Recently Loukitcheva et al. (2006) compared these models to observations done with the BerkeleyIllinois-Maryland Array (BIMA, White et al. 2006). The major problem of observations at millimetre wavelengths is the generally poor spatial resolution which renders granular scales so far unaccessible - even with the BIMA array with its 10 antennae. The situation will substantially improve with the next generation of large interferometric arrays, e.g., the Atacama Large Millimeter Array (ALMA) which will be fully operational in 2012 (e.g. Beasley et al. 2006). This instrument will provide high spatial and temporal resolution, finally allowing to observe the small-scale structure of the solar chromosphere in detail.

Here we use three-dimensional radiation hydrodynamics simulations to synthesise the continuum intensity at millimetre wavelengths from $0.3 \mathrm{~mm}$ to $9 \mathrm{~mm}$ (see Wedemeyer-Böhm et al. 2005 , for a precursory study). Since the simulations do only include weak magnetic fields or no field, the present analysis refers to internetwork regions only.

In Sect. 2 some instruments, that are potentially interesting for solar observations, are introduced. The numerical simulations and the method of producing synthetic intensity images are described in Sects. 3 and 4, respectively. The results are presented in Sect. 5, followed by discussion and conclusions in Sects. 6 and 7.

\section{Instruments}

The instruments addressed in this section can (optionally) be used as interferometers and are potentially interesting for solar observations. Although the angular resolution of an interferometer can be very large depending on the maximum baseline (connecting two individual antennae), there is an effective maximum resolution on which highly reliable images of objects such as the Sun, with complicated emission filling the whole primary beam of each antenna, can be made. Being a dynamic object the amount of received data constraining a snapshot image equals twice the number of baselines (real and imaginary parts of each visibility being counted separately). The number of unknowns for the image construction, however, is equal to the number of independent synthesised beam areas within the primary beam. Reliable imaging requires that there are more data constraints than there are unknowns. This condition defines the effective resolution of reliable images for the interferometer. Here we estimate this resolution by assuming a homogeneous distribution of the synthesised beams ("image elements") over the primary beam area (the field of view, FOV). The number of those elements is equal to the number of baselines. An array with $N_{\mathrm{a}}$ antennae gives a maximum of

$N_{\mathrm{b}}=\frac{N_{\mathrm{a}}\left(N_{\mathrm{a}}-1\right)}{2}$

possible baselines. Depending on technical details of the array, only a subset might be realised. As such details might change, we always refer to the maximum number in this work. Also note that a necessarily finite number of base lines limits the number of resolution elements. A finite number of baselines, however, can only provide an incomplete coverage of the $u-v$-plane (spatial Fourier space), making it difficult to determine the effective spatial resolution. For our estimate of the effective spatial resolution $\Delta \alpha$ we assume full $u-v$ coverage in the limit of a large number of baselines. We finally derive the relation

$$
(\Delta \alpha)_{\lambda} \approx \frac{d_{\lambda}}{\sqrt{2 N_{\mathrm{b}}}}=\frac{d_{\lambda}}{\sqrt{N_{\mathrm{a}}\left(N_{\mathrm{a}}-1\right)}} .
$$

The primary beam size $d$ and thus the resolution, too, depends proportionally on wavelength $\lambda$. Note that our estimate is not a hard limit. Reliable images should be possible at significantly higher resolution, using the known positivity of the image and other "a priori" constraints. The technique of Multi-Frequency Synthesis (MFS) uses the frequency-dependence of the $u$ - $v$ coverage, resulting in a larger number of data constraints (Conway et al. 1990). It has thus the potential of significantly increasing the effective resolution of reliable images. However, detailed imaging simulations would be needed to quantify the achievable resolution (Conway 2007).

The list of interferometers in Sects. 2.1-2.5 contains characteristic properties that are relevant for the discussion in Sect. 6.6. Some properties like the FOV, however, are not easily expressed with a single number - in particular for the heterogeneous arrays CARMA, FASR, and RAINBOW since the different dish diameters of the individual antenna types result in different (wavelength-dependent) primary beam sizes. Please note that the description of the interferometers relies on information available in the literature and in the internet and is only thought to give a broad overview. Technical details might differ slightly in practice.

\subsection{ALMA}

Among a large range of issues important to modern astronomy, the Atacama Large Millimeter Array (ALMA) will also be used for observations of the Sun. While its single-dish predecessor APEX (Atacama Pathfinder Experiment) has already been installed and delivers first scientific results, the construction of the array started next to the APEX site on a plateau at $5000 \mathrm{~m}$ altitude in the Chilean Andes. The science verification phase will most likely start in late 2009, followed by an early science stage from 2010, and finally full operation in 2012. All details given in this section refer to Bastian (2002), Brown et al. (2004), more recently Beasley et al. (2006) and the ALMA web pages (e.g., http:// www.alma.info, http://www.alma.nrao.edu, http:// www.eso.org/projects/alma/). See also Escoffier et al. (2007).

The main array will consist of 50 antennae with a diameter of $12 \mathrm{~m}$ in an adjustable configuration ranging from a compact size of $150 \mathrm{~m}$ to a maximum base length of $\sim 18.5 \mathrm{~km}$. It is supplemented with the (semi-independent) Atacama Compact Array (ACA) with 12 antennae with 7 m diameter and $412 \mathrm{~m}$-antennae. Each ALMA antenna will be equipped with receivers which cover up to ten frequency bands. Initially only the frequency bands in the range from $84 \mathrm{GHz}$ to $373 \mathrm{GHz}$ will operate (see Table 1). This range corresponds to wavelengths from $3.57 \mathrm{~mm}$ to $0.80 \mathrm{~mm}$. The correlator can subdivide the frequency bands into a large number of spectral channels $(\sim 16000)$ although lower spectral resolution can easily be achieved by reconfiguring the correlator or by post-correlation spectral averaging of the data. A minimum integration time of $16 \mathrm{~ms}$ might be possible whereas switching between different bands will take $\sim 1.5 \mathrm{~s}$.

The field of view is defined by the primary beam size of an ALMA antenna, being $21^{\prime \prime}$ at $\lambda=1 \mathrm{~mm}$. That is sufficient to 
Table 1. Frequency bands of ALMA that will be installed first and corresponding values for the expected primary beam size diameter $d_{\lambda}$ and the estimated spatial resolution $(\Delta \alpha)_{\lambda}$ for images of maximum reliability (see text for details). A total number of 50 antennae is assumed.

\begin{tabular}{ccccc}
\hline \hline Band & $v[\mathrm{GHz}]$ & $\lambda[\mathrm{mm}]$ & $d_{\lambda}\left[^{\prime \prime}\right]$ & $(\Delta \alpha)_{\lambda}\left[^{\prime \prime}\right]$ \\
\hline 3 & $84-116$ & $3.57-2.58$ & $75-54$ & $1.51-1.10$ \\
6 & $211-275$ & $1.4-1.09$ & $30-23$ & $0.60-0.46$ \\
7 & $275-373$ & $1.09-0.80$ & $23-17$ & $0.46-0.34$ \\
\hline
\end{tabular}

observe the interior of an internetwork region. In interferometric mode ALMA will provide an angular resolution of 0 ". 015 to 1 '.4, depending on antenna configuration. The angular resolution corresponds to $\sim 10 \mathrm{~km}$ to $\sim 1000 \mathrm{~km}$ on the Sun. The number of baselines is 1225 for the main array with 50 large antennae (see Eq. (1)). With Eq. (2) we estimate the effective spatial resolution for images of maximum reliability, $(\Delta \alpha)_{\lambda}$, to be of the order of $\sim 0$ ". 42 at a wavelength of $1 \mathrm{~mm}$. The resolution for $0.3 \mathrm{~mm}$ and $3 \mathrm{~mm}$ would be 0.13 and $1{ }^{\prime \prime} .27$, resp. (see also Table 1). A number of 64 antennae, as originally planned, would result in 2016 baselines. The total number of baselines, when including ACA, could be up to $2016+120$, reaching an effective resolution of $\sim 0$ ". 32 at $\lambda=1 \mathrm{~mm}$.

\subsection{CARMA}

The two millimetre arrays of Owens Valley Radio Observatory (OVRO) and of the Berkeley-Illinois-Maryland Association (BIMA) were merged to form the Combined Array for Research in Millimeter-wave Astronomy (CARMA) at Cedar Flat, California (Woody et al. 2004; Beasley \& Vogel 2003). This heterogeneous array consists of six $10.4 \mathrm{~m}$ antennae (OVRO) and ten $6.1 \mathrm{~m}$ antennae (BIMA), resulting in 105 baselines. There are receivers available for $115 \mathrm{GHz}(2.6 \mathrm{~mm})$ and $230 \mathrm{GHz}$ $(1.3 \mathrm{~mm})$. CARMA will be supplemented with a subarray consisting of $83.5 \mathrm{~m}$ antennae from the Sunyaev-Zeldovich Array (SZA) for observations at $115 \mathrm{GHz}$ but also $35 \mathrm{GHz}(\sim 1 \mathrm{~cm})$ with a total of 276 possible baselines. Different array configurations with spacing from down to $5 \mathrm{~m}$ to $1.9 \mathrm{~km}$ are possible. An angular resolution of 0 ". 1 can be reached (for the $230 \mathrm{GHz}$ A-array). See Woody et al. (2004), Beasley \& Vogel (2003), and http://www . mmarray . org for more information.

\subsection{EVLA}

During the first project phase the Expanded Very Large Array (EVLA, see http://wWw.aoc.nrao.edu/evla) consists of the 27 VLA antennae with primary reflector diameters of $25 \mathrm{~m}$, providing 351 baselines. In the second phase the array will be supplemented with eight new antennae, resulting in baselines of up to $350 \mathrm{~km}$. The array will then have a very high angular resolution of 0 ".004 at $\lambda=6 \mathrm{~mm}$. The accessible frequencies range from $1.0 \mathrm{GHz}$ to $50 \mathrm{GHz}$, corresponding to $30 \mathrm{~cm}$ to $6 \mathrm{~mm}$. The correlator will provide some thousand frequency channels.

\subsection{FASR}

The Frequency-Agile Solar Radiotelescope (FASR, see http://wWw . ovsa.njit. edu/fasr) will combine three types of antenna, necessary to cover the large range from $30 \mathrm{MHz}$ $(\lambda \approx 10 \mathrm{~m})$ to $30 \mathrm{GHz}(\lambda \approx 1 \mathrm{~cm})$. The sub-array for the high frequencies will consist of $\sim 100$ antennae of $2 \mathrm{~m}$ diameter each, setting up roughly 5000 baselines. The maximum antenna
Table 2. Numerical models used for this study: publication describing the individual models in more detail, short description, code used for intensity synthesis, number of time steps $n_{\mathrm{t}}$, and heliocentric angles $(\mu=\cos \theta)$.

\begin{tabular}{clllrc}
\hline \hline Model & Pub. & Description & $\begin{array}{l}\text { Synthesis } \\
\text { code }\end{array}$ & $n_{\mathrm{t}}$ & $\mu$ \\
\hline $\mathrm{A}$ & W04 & non-magnetic & Linfor3D & 60 & $0.2-1.0$ \\
$\mathrm{~B}$ & $\mathrm{~S} 06$ & $\begin{array}{l}\text { magnetic, } \\
\left|B_{0}\right|=10 \mathrm{G} \\
\text { non-equilibrium } \\
\text { H ionisation }\end{array}$ & Linfor3D & 1 & 1.0 \\
$\mathrm{C}$ & LW06 & 1 & 1.0 \\
\hline
\end{tabular}

spacing will be $6 \mathrm{~km}$. At $30 \mathrm{GHz}$ an angular resolution of $\sim 0$ ". 66 can be reached. FASR will produce image sequences including polarisation information with a time resolution of less than $0.1 \mathrm{~s}$. The construction is expected to be completed in 2010 .

\subsection{RAINBOW}

The six transportable $10 \mathrm{~m}$-antennae of the Nobeyama Millimeter Array have been linked to the local $45 \mathrm{~m}$-antenna to form the RAINBOW interferometer (http://www.nro.nao.ac.jp). The maximum baseline length is of the order of $400 \mathrm{~m}$. RAINBOW can access wavelengths from $1.3 \mathrm{~mm}$ to $3.5 \mathrm{~mm}$.

\section{Numerical models}

The numerical 3-D models used here are calculated with the radiation hydrodynamics code $\mathrm{CO}^{5} \mathrm{BOLD}$ (Freytag et al. 2002). Most of the study refers to the non-magnetic model by Wedemeyer et al. (2004, hereafter W04, model A), whereas only one snapshot is taken each from the models by Schaffenberger et al. (2006, hereafter S06, model B) and Leenaarts \& Wedemeyer-Böhm (2006b, hereafter LW06, model C). The models are described below (see also Table 2).

For all models a grey, i.e., frequency-independent radiative transfer is used. The lateral boundary conditions are periodic in all variables, whereas the lower boundary is "open" in the sense that the fluid can freely flow in and out of the computational domain under the condition of vanishing total mass flux. The specific entropy of the inflowing mass is fixed to a value previously determined so as to yield solar radiative flux at the upper boundary. The upper boundary is "transmitting" in the hydrodynamics case (models A, C) but "closed" for the MHD model B, i.e., reflecting boundaries are applied to the vertical velocity, while stress-free conditions are in effect for the horizontal velocities.

Please note that we define the term photosphere as the layer between $0 \mathrm{~km}$ and $500 \mathrm{~km}$ in model coordinates, and the term chromosphere as the layer above. The origin of the geometric height scale is chosen to match the temporally and horizontally averaged Rosseland optical depth unity for each model individually. The computational time step is around 0.1 to $0.2 \mathrm{~s}$ in hydrodynamics case (models A, C) and an order of magnitude smaller for the MHD model B.

The utilised models still have short-comings concerning the energy balance of the chromosphere but nevertheless can give a first idea of the small-scale structure and dynamics of this layer. Refer to Sect. 6.1 for a discussion of the limitations of the modelling. 

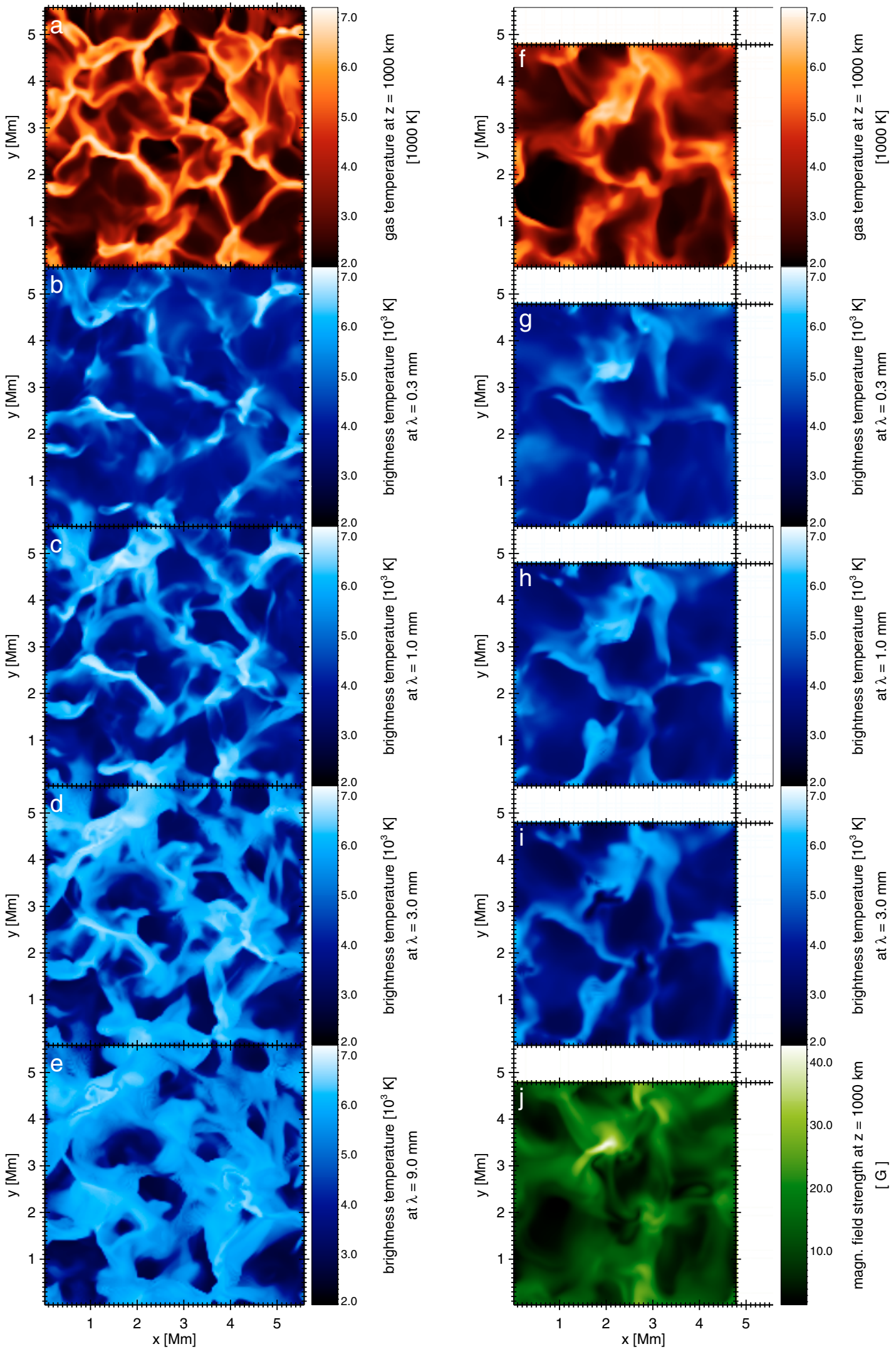

Fig. 1. Horizontal maps from the non-magnetic model A (top) and the somewhat smaller MHD model B (bottom). Panels a) and f) show the gas temperature at a geometrical height of $z=1000 \mathrm{~km}$. In panels $\mathbf{b}-\mathbf{e}$ ) the brightness temperature for model A is displayed for wavelengths of $0.3 \mathrm{~mm}$, $1 \mathrm{~mm}, 3 \mathrm{~mm}$, and $9 \mathrm{~mm}$, respectively. The brightness temperature is also shown for model B at wavelengths of $\mathbf{g}) 0.3 \mathrm{~mm}, \mathbf{h}) 1 \mathrm{~mm}$, and i) $3 \mathrm{~mm}$. The magnetic field strength $|\boldsymbol{B}|$ at a height of $z=1000 \mathrm{~km}$ in model B is plotted in panel $\mathbf{j}$ ). The quantities are colour-coded as indicated by the legend next to each panel. Please note that the intensity contribution functions partly exceed the upper boundaries of the models for the panels $\mathbf{e}$ ) and i), causing artefacts in the resulting brightness temperature maps. For the same reason $\lambda=9 \mathrm{~mm}$ is not shown for model B. 


\subsection{Field-free hydrodynamic model}

Model A (W04) does not include magnetic fields. The computational domain consists of $140 \times 140$ grid cells in horizontal $(x, y)$ and 200 in vertical direction. While the horizontal resolution is constantly $40 \mathrm{~km}$, the vertical resolution varies from $46 \mathrm{~km}$ at the bottom in the upper convection zone at $z=-1400 \mathrm{~km}$ to $12 \mathrm{~km}$ for all layers above $z=-270 \mathrm{~km}$. The top of the model is located at a height of $z=1710 \mathrm{~km}$ in the middle chromosphere. The horizontal extent is $5600 \mathrm{~km}$, corresponding to an angle of $7^{\prime \prime} .7$ in ground-based observations. For the analysis presented here, we use a partial sequence with a time increment of $10 \mathrm{~s}$ and a duration of $600 \mathrm{~s}$. The model chromosphere is characterised by a mesh-like pattern of hot shock fronts and cool post-shock regions inbetween (see Fig. 1a).

\subsection{Magnetohydrodynamic model}

Model B (S06) includes a weak magnetic field with an average flux density of $10 \mathrm{G}$. The computational domain is somewhat smaller than for model A. It extends over a height range of $2800 \mathrm{~km}$ of which $1400 \mathrm{~km}$ reach below the mean surface of optical depth unity (the same as for model A) and $1400 \mathrm{~km}$ above it. The horizontal extent is $4800 \mathrm{~km} \times 4800 \mathrm{~km}$. With $120^{3}$ grid cells, the spatial resolution in the horizontal direction is constantly $40 \mathrm{~km}$, whereas it varies between 50 and $20 \mathrm{~km}$ in the vertical direction, thus accounting for the varying scale heights in the atmosphere. The initial model has a homogeneous, vertical, unipolar magnetic field with a flux density of $10 \mathrm{G}$ which is superposed on a previously computed, relaxed model of thermal convection very similar to model A. In the course of the simulation the convective flows advect the magnetic field towards the intergranular lanes where stronger flux concentrations ("flux tubes") build up. In contrast, the model chromosphere is characterised by a more homogeneous but more rapidly varying field distribution (see Fig. 1j). The gas temperature still is similar to the one in model A (see Fig. 1f).

\subsection{Model with non-equilibrium hydrogen ionisation}

Model C (LW06, see also Leenaarts \& Wedemeyer-Böhm 2006a) is identical to model A with respect to the numerical grid, the radiative transfer, and the hydrodynamics solver. It also does not contain magnetic fields. The only difference compared to model $\mathrm{A}$ is that the hydrogen ionisation is not treated in LTE but in non-equilibrium (non-LTE) by solving the time-dependent rate equations for a six level model atom with fixed radiative rates. Hydrogen is treated as minor species so far, i.e. the nonequilibrium hydrogen ionisation has no back-coupling on the equation of state and on the opacities. The gas temperature distribution is thus (statistically) the same as for model A. In contrast to model A, however, the code outputs the electron densities and hydrogen level populations for the LTE case and the NLTE case for each grid cell for model $\mathrm{C}$. The electron density contribution of hydrogen directly results from the non-LTE computation, whereas the contributions of other chemical species are treated in LTE (see LW06 for details). The electron density in the model chromosphere varies much less in non-LTE compared to LTE.

\section{Intensity synthesis}

Continuum radiation at (sub-)mm wavelengths is mainly due to thermal free-free emission and originates from the chromosphere and upper photosphere. The opacity is mostly due to free-free processes (interaction of ions and free electrons), including $\mathrm{H}^{-}$free-free (interaction of neutral hydrogen atoms and free electrons). Owing to the large wavelength and thus small frequency the condition

$h v \ll k_{\mathrm{B}} T_{\text {gas }}$

is fulfilled so that the Rayleigh-Jeans approximation can be used. According to Rybicki \& Lightman (2004, see Eq. (5.19a)) and Mihalas Mihalas (1978, cf. p. 102), the opacity coefficient for thermal free-free bremsstrahlung (ion-electron interaction) can then be written as

$\kappa_{\mathrm{ff}, v} \propto \frac{n_{\mathrm{e}} n_{\mathrm{I}}}{v^{2} T_{\mathrm{gas}}^{3 / 2}}$

where $n_{\mathrm{e}}$ and $n_{\mathrm{I}}$ are the number densities of electrons and ions, resp., and $v$ and $T_{\text {gas }}$ are frequency and gas temperature. In particular the dependence of $\kappa_{\mathrm{ff}}$ on $n_{\mathrm{e}}$ and $v^{-2}$ is of importance for the optical depth at wavelengths around $1 \mathrm{~mm}$ and thus of particular interest for the study presented here. The free-free processes are due to collisions with electrons and depend on the local thermodynamic state of the electrons. The ratio of emission to absorption processes is thus in local thermodynamic equilibrium (LTE), and the source function is Planckian. Another consequence of Eq. (3) is that the contribution to the emergent intensity / brightness temperature at (sub-)mm wavelengths is linearly related to the local gas temperature in the contributing height range. The electron densities, however, can deviate from the LTE values. That is caused by (i) ionisation by a non-Planckian radiation field, in particular in the Balmer continuum, and (ii) the long recombination timescales that hinder the hydrogen ionisation degree to follow the faster dynamic changes of the atmospheric conditions (Carlsson \& Stein 2002, LW06). This deviation from equilibrium has an effect on the optical depth and thus on the effective formation height of the radiation via the absorption coefficient (see Eq. (4)). For this first qualitative study, we neglect the resulting effect on the opacity for the intensity synthesis from model A and B but investigate the effect for model C (see Sects. 5.2 and 6).

For the radiative transfer calculations for model A and B we use Linfor3D, a 3D LTE spectrum synthesis code developed by M. Steffen and H.-G. Ludwig, which is originally based on the Kiel code LINFOR/LINLTE (see http:// www.aip.de/ mst/linfor3D_main.html). Electron densities are calculated under the assumption of LTE. For model C we use the RH code by Uitenbroek (2000). We calculate a snapshot with LTE electron densities but also with the non-equilibrium electron densities, which are a direct result of the simulation with non-equilibrium hydrogen ionisation (see Sect. 3.3).

Continuum intensity images are calculated at the four wavelengths $0.3 \mathrm{~mm}(\sim 1000 \mathrm{GHz}), 1 \mathrm{~mm}(\sim 300 \mathrm{GHz}), 3 \mathrm{~mm}$ $(\sim 100 \mathrm{GHz})$, and $9 \mathrm{~mm}(\sim 33 \mathrm{GHz})$. The heliocentric angle is hereafter referred to as the inclination angle $\theta$ and its corresponding $\operatorname{cosine} \mu=\cos \theta$. For model $\mathrm{A}$ we consider five different positions on the solar disk from $\mu=1.0$ (disk-centre) to 0.2 (near limb) with an increment of $\Delta \mu=0.2$. With a number of 60 snapshots this results in a total of $60 \times 4 \times 5$ intensity maps. For model B and also the LTE and NLTE case for model C only disk-centre maps for one snapshots are computed, giving 4 intensity maps in each case.

For convenience, intensities $I_{\lambda}$ in units of $\operatorname{erg} \mathrm{cm}^{-2} \mathrm{~s}^{-1} \AA^{-1} \mathrm{sr}^{-1}$ (as output by LINFOR3D) are converted 

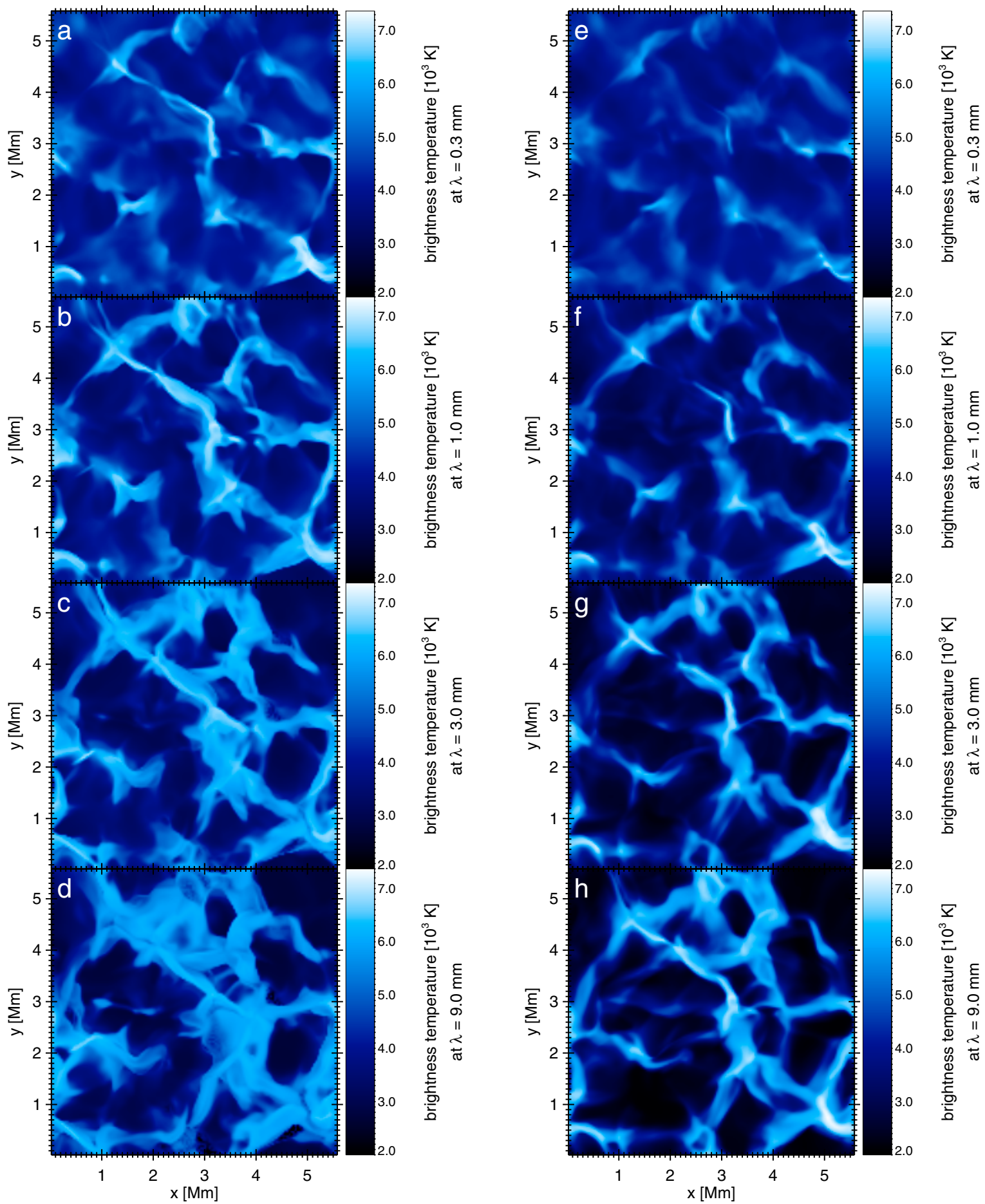

Fig. 2. Horizontal maps from the non-magnetic model $\mathrm{C}$ with electron densities treated in LTE (top) and with the time-dependent non-equilibrium electron densities (NLTE, bottom), which result from the simulation. The wavelengths from top to bottom are $0.3 \mathrm{~mm}, 1 \mathrm{~mm}, 3 \mathrm{~mm}$, and $9 \mathrm{~mm}$, respectively. Please note that the average intensity contribution function for panel d partly exceeds the upper boundary of the model.

to brightness temperature $T_{\mathrm{b}}$ via a relation derived from the Kirchhoff-Planck function in the limit of Eq. (3):

$T_{\mathrm{b}}=\frac{\lambda^{4}}{2 k_{\mathrm{B}} c} I_{\lambda}$.

Here, $\lambda, k_{\mathrm{B}}$, and $c$ are the wavelength, the Boltzmann constant and the speed of light, respectively. The intensity contribution function $C$ along a vertical view angle $(\mu=1.0)$ is defined as

$C(z)=\kappa(z) \rho(z) \mathcal{B}_{\lambda}(z) \mathrm{e}^{-\tau_{\lambda}(z)}$.
The above quantities are opacity $\kappa$ (extinction coefficient per mass unit), density $\rho$, Planck function $\mathcal{B}_{\lambda}$, and optical depth $\tau_{\lambda}$ which are functions of geometrical height $z$. Integration of the contribution functions over all heights yields the emergent intensity.

Examples for the resulting intensity maps are shown in Fig. 1 for models A and B at disk-centre, in Fig. 2 for model C, and for different inclination angles for model A in Fig. 9. 


\section{Results}

\subsection{Brightness temperature distribution}

The intensity or brightness temperature maps for the different wavelengths all exhibit the complex pattern of hot/bright filamentary structures and cool/dark regions inbetween that is already seen in the gas temperature cuts through the model chromospheres. See Fig. 1 for an example of a time step from model A and model B, and Fig. 2 for the LTE and NLTE cases from model $\mathrm{C}$, respectively. Nevertheless, there are differences in the brightness temperature distribution which we quantify here by means of the horizontal and, in case of model A, temporal average $\left\langle T_{\mathrm{b}}\right\rangle$ and the relative intensity contrast

$\frac{\delta I_{\mathrm{rms}}}{\langle I\rangle}=\frac{\delta T_{\mathrm{b}, \mathrm{rms}}}{\left\langle T_{\mathrm{b}}\right\rangle}=\frac{\sqrt{\left\langle T_{\mathrm{b}}^{2}\right\rangle-\left\langle T_{\mathrm{b}}\right\rangle^{2}}}{\left\langle T_{\mathrm{b}}\right\rangle}$,

where $\delta T_{\mathrm{b}, \mathrm{rms}}$ is the brightness temperature fluctuation. The values are listed in Table 3 for all models, wavelengths, and inclination angles. As we shall see later in more detail, the longest wavelength is only of limited meaning in the present study since the formation height range partly exceeds the vertical extent of the numerical models.

The average brightness temperature for model A increases with wavelength $\lambda$ which is due to the corresponding change in effective formation height (see Sect. 5.2). The same effect is found when going from disk-centre towards the limb, i.e. with increasing inclination angle $\theta$. The LTE case for model $\mathrm{C}$ behaves in a similar way whereas the average temperatures decrease with wavelength for the corresponding NLTE case and also model B.

The absolute brightness temperature fluctuation $\delta T_{\mathrm{b}, \mathrm{rms}}$ tends to increase with wavelength at or close to disk-centre while this trend reverses closer to the limb. For model A this means an increase of the rms temperature amplitude from $\sim 800 \mathrm{~K}$ to $\sim 1100-1200 \mathrm{~K}$. The effect is more pronounced for models B and $\mathrm{C}$. The relative brightness temperature contrast $\delta T_{\mathrm{b}, \mathrm{rms}} /\left\langle T_{\mathrm{b}}\right\rangle$ depends in a similar way on wavelength and inclination angle. Despite the large temperature fluctuations in the layers apparently sampled by the long wavelengths (see Sect. 5.2), the contrast reduces to only $\sim 12 \%$ for $\lambda=9 \mathrm{~mm}$ close to the limb. In contrast, the maps from model A at disk-centre show contrasts of up to $24 \%$ with exception of the shortest wavelength that provides $19 \%$ at maximum. Model B has much larger fluctuations with contrasts from $27 \%$ to $34 \%$ implying a detectable influence of the weak magnetic field on the gas and brightness temperature distribution. The contrast in the snapshot with LTE electron densities from model $\mathrm{C}$ is similar to model $\mathrm{A}$, although with a larger spread with wavelength. This can be explained by the fact that the contrast for model A relies on 60 snapshots but only on a single one for model $\mathrm{C}$. The trend of the contrast to increase with wavelengt is even more pronounced in the NLTE case. The more homogeneous electron densities reduce the contrast at $\lambda=0.3 \mathrm{~mm}$ to only $\sim 13 \%$ but increase it for the other wavelengths. The different behaviour of the shortest wavelength is related to the fact that the bulk of emission originates predominantly from layers where the amplitudes of the propagating shock waves are still relatively small (upper photosphere) - in contrast to the other wavelengths which sample higher layers (see Sect. 5.2).

The less corrugated surface of equal optical depth results in less contributions from layers with higher gas temperature fluctuations. As a consequence the NLTE case provides a cleaner measure of the layer near the classical temperature minimum at

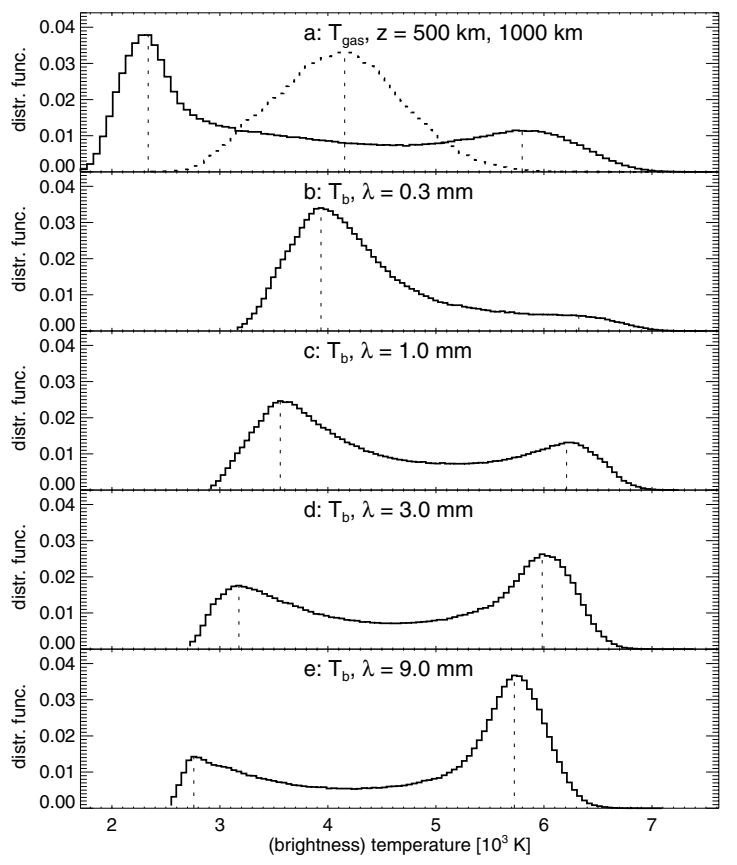

Fig. 3. Temperature distribution in model A: Histograms for a) gas temperature in horizontal slices at geometrical heights of $z=500 \mathrm{~km}$ (dotted) and $z=1000 \mathrm{~km}$ (solid), and b-e) brightness temperature at wavelengths of $0.3 \mathrm{~mm}, 1 \mathrm{~mm}, 3 \mathrm{~mm}$, and $9 \mathrm{~mm}$, respectively (all diskcentre). All time steps of the analysed 3-D model sequence are taken into account. The vertical dashed lines mark the positions of the individual hot and cool peaks.

the top of the model photosphere where the temperature fluctuations are small. At the longer wavelengths, only the shockpatterned chromosphere is sampled in the NLTE case and also in LTE there are significant contributions from that layer. In contrast to the region of low amplitude around the classical temperature minimum the chromosphere shows a similar pattern of hot shock fronts and cool post-shock regions for all heights above $z \sim 700 \mathrm{~km}$. In LTE, the optical depth roughly follows the temperature gradients so that the chromosphere is sampled on corrugated surface of equal optical depth. The corresponding surfaces in NLTE vary much less in height and thus cut through the temperature fluctuations instead of following them, explaining the higher contrast in NLTE compared to LTE.

The difference in intensity distribution is shown more detailed in form of histograms in Fig. 3 for the four wavelengths (all for disk-centre only) and for gas temperature at geometric heights of $z=500 \mathrm{~km}$ and $1000 \mathrm{~km}$ (uppermost panel) for model A. As described in more detail by W04, the distribution of gas temperature exhibits a cool and a hot component with an intermediate range. The two components are due to a cool background and hot shock fronts. W04 also provide histograms for other heights (see Fig. 7 therein). The histograms for continuum intensity show two components, too, although with varying amplitudes. At $\lambda=0.3 \mathrm{~mm}$ a strong low-intensity component is visible whereas it is hard to define a high-value peak at all. The distribution for $\lambda=1 \mathrm{~mm}$ is most similar to the gas temperature histogram at $z=1000 \mathrm{~km}$ with peaks in almost the right proportion. In contrast, the high-intensity peak is more pronounced than the low-value component in case of the longer wavelengths $(\lambda=3 \mathrm{~mm}, 9 \mathrm{~mm})$. The differences between the wavelengths can be understood if one considers the different height ranges that contribute to the continuum intensity (see Sect. 5.2). The 
Table 3. Average brightness temperature $\left\langle T_{\mathrm{b}}\right\rangle$, rms variation $\delta T_{\mathrm{b}, \mathrm{rms}}$, and brightness temperature contrast $\delta T_{\mathrm{b}, \mathrm{rms}} /\left\langle T_{\mathrm{b}}\right\rangle$ for all wavelengths $\lambda$ and disk-positions $\mu$ derived from all snapshots. Due to the linear conversion at given $\lambda$ the values can also be interpreted as intensity contrast $\delta I_{\text {rms }} /\langle I\rangle$. In some cases the corresponding average contribution function partly exceeds the upper boundary of the model. The missing contribution is in the range of $0.5 \%$ to $2 \%$ for data marked with ${ }^{*}$ and $>2 \%$ for data marked with ${ }^{* *}$.

\begin{tabular}{|c|c|c|c|c|c|c|c|c|c|c|c|c|c|}
\hline & & & & $b_{b}>[K]$ & & & & $\mathrm{ms}[\mathrm{K}]$ & & & & $\frac{\frac{b, \mathrm{rms}}{\left.T_{\mathrm{b}}\right\rangle}}{2}$ & \\
\hline \multirow[t]{2}{*}{ Model } & \multirow[t]{2}{*}{$\mu$} & \multicolumn{4}{|c|}{$\lambda[\mathrm{mm}]$} & \multicolumn{4}{|c|}{$\lambda[\mathrm{mm}]$} & \multicolumn{4}{|c|}{$\lambda[\mathrm{mm}]$} \\
\hline & & 0.3 & 1.0 & 3.0 & 9.0 & 0.3 & 1.0 & 3.0 & 9.0 & 0.3 & 1.0 & 3.0 & 9.0 \\
\hline \multirow[t]{5}{*}{$\mathrm{A}$} & 0.2 & 5033 & 5339 & 5397 & 5332 & 820 & 726 & 642 & 587 & 0.163 & 0.136 & 0.119 & 0.110 \\
\hline & 0.4 & 4758 & 5145 & $5340^{*}$ & $5323^{*}$ & 914 & 1019 & $908^{*}$ & $777^{*}$ & 0.192 & 0.198 & $0.170^{*}$ & $0.146^{*}$ \\
\hline & 0.6 & 4605 & 4918 & $5149^{*}$ & $5178^{* *}$ & 884 & 1102 & $1076^{*}$ & $968^{* *}$ & 0.192 & 0.224 & $0.209^{*}$ & $0.187^{* *}$ \\
\hline & 0.8 & 4530 & 4770 & $4983^{*}$ & $5031^{* *}$ & 852 & 1111 & $1156^{*}$ & $1092^{* *}$ & 0.188 & 0.233 & $0.232^{*}$ & $0.217^{* *}$ \\
\hline & 1.0 & 4496 & 4684 & $4867^{*}$ & $4913^{* *}$ & 814 & 1105 & $1188^{*}$ & $1155^{* *}$ & 0.181 & 0.236 & $0.244^{*}$ & $0.235^{* *}$ \\
\hline $\mathrm{B}$ & 1.0 & 5074 & 5001 & $4860^{* *}$ & $3569^{\text {** }}$ & 1380 & 1505 & $1623^{* *}$ & $1213^{* *}$ & 0.272 & 0.301 & $0.334^{* *}$ & $0.340^{* *}$ \\
\hline \multirow[t]{3}{*}{$\mathrm{C}$} & \multicolumn{13}{|c|}{ LTE electron densities: } \\
\hline & 1.0 & $\begin{array}{l}4462 \\
\text { NLTE }\end{array}$ & 4476 & $\begin{array}{l}4518^{*} \\
\text { densities. }\end{array}$ & $4517^{* *}$ & 718 & 998 & $1170^{*}$ & $1229^{* *}$ & 0.161 & 0.223 & $0.259^{*}$ & $0.272^{* *}$ \\
\hline & 1.0 & 4213 & 3935 & 3768 & 3910 & 527 & 933 & 1266 & 1482 & 0.125 & 0.237 & 0.336 & 0.379 \\
\hline
\end{tabular}

low-value peak in gas temperature at $z=1000 \mathrm{~km}$ is at lower values than for the brightness temperature distributions. A linear conversion between both temperatures due to the validity of LTE (see Sect. 4) should produce identical distributions for gas temperature and corresponding brightness temperature at the same height. The differences in Fig. 3, however, illustrate the influence of the extended formation height ranges (see Sect. 5.2) which effectively mixes contributions from different layers instead of sampling the temperature at a fixed geometrical height, as it is the case for the displayed gas temperature maps.

\subsection{Contribution functions and formation heights}

In the following we describe the effective formation heights by means of $z_{\operatorname{maxc}}$, the height of the maximum of the horizontally (and temporally) averaged contribution functions, and by $z_{\mathrm{cc}}$, the height of the centroid of the (spatially resolved) contribution functions, where $z_{\mathrm{cc}}$ is defined as

$z_{\mathrm{cc}}=\frac{\int z^{\prime} \cdot C\left(z^{\prime}\right) \mathrm{d} z^{\prime}}{\int C\left(z^{\prime}\right) \mathrm{d} z^{\prime}}$.

All available horizontal positions (and time steps) are used for the calculation of the average $\left\langle z_{\mathrm{cc}}\right\rangle$ and the variation $\delta\left(z_{\mathrm{cc}}\right)_{\mathrm{rms}}$. The results are summarised in Table 4 . The horizontally and temporally averaged contribution functions for model A are shown in Fig. 4 for all wavelengths and inclination angels. For diskcentre $(\mu=1.0)$, the continuum intensity at $0.3 \mathrm{~mm}$ originates mainly from layers near the classical temperature minimum region with a contribution peak at a height of $z_{\operatorname{maxc}}=481 \mathrm{~km}$. Additionally, there are small contributions from the low chromosphere. The picture is in principle the same for a wavelength of $1 \mathrm{~mm}$, although the peak is located somewhat higher at a height of $588 \mathrm{~km}$ and the chromospheric contribution is larger. Hence, these both wavelengths map mainly the thermal structure of the top of the photosphere and the low chromosphere. Next to a maximum at $z_{\operatorname{maxc}}=695 \mathrm{~km}$ the emission at $\lambda=3 \mathrm{~mm}$ originates also from an extended height range in the chromosphere with a subtle secondary maximum at $1120 \mathrm{~km}$. At $\lambda=9 \mathrm{~mm}$ all chromospheric heights contribute almost equally to the intensity with a maximum at $1348 \mathrm{~km}$ and a less pronounced, slightly smaller peak (0.98) at a height of $789 \mathrm{~km}$ which might be compared to the peaks for the shorter wavelengths. The contribution

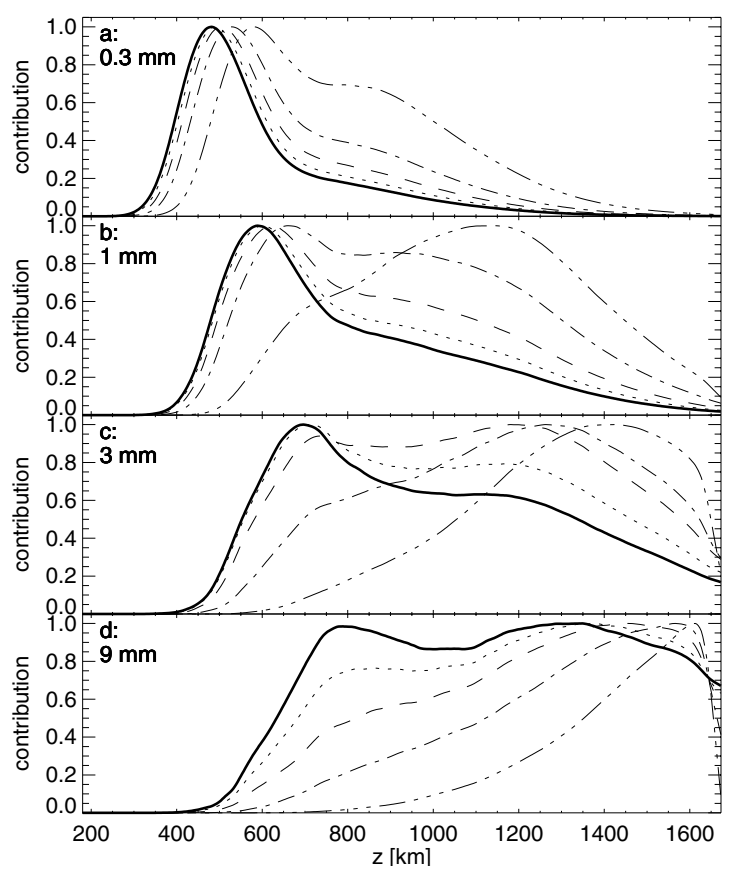

Fig. 4. Normalised contribution functions for continuum intensity on the geometrical height scale calculated with Linfor3D from the model by W04 (average over all horizontal positions and time steps in the analysed data sample) at wavelengths of a) $0.3 \mathrm{~mm}$, b) $1 \mathrm{~mm}$, c) $3 \mathrm{~mm}$, and d) $9 \mathrm{~mm}$ for $\mu=1.0$ (thick solid), $\mu=0.8$ (dotted), $\mu=0.6$ (dashed), $\mu=0.4$ (dot-dashed), $\mu=0.2$ (triple-dot-dashed). The assumption of LTE was made for the calculations.

function implies that there would still be significant emission from layers above the computational domain. Therefore the intensity images for $\lambda=9 \mathrm{~mm}$, as shown in Fig. 1, and also the results derived for this wavelength are incomplete to some extent and thus of only limited significance. According to Loukitcheva et al. (2004), emission at $\lambda \geq 8 \mathrm{~mm}$ does even originate from the transition region which is not included in the models used here. Consequently, a more extended model would be necessary for correctly modelling emission at long wavelengths.

Owing to the complicated thermal structure and the corresponding LTE electron density, a large height range is involved in emitting radiation at a certain wavelength, causing the 
Table 4. Heights of maximum contribution $z_{\operatorname{maxc}}$ (see Fig. 4), average and variation of the heights of the centroids of the spatially resolved contribution functions, $\left\langle z_{\mathrm{cc}}\right\rangle$ and $\delta\left(z_{\mathrm{cc}}\right)_{\mathrm{rms}}$, resp., for all wavelengths $\lambda$ and disk-positions $\mu$ derived from all snapshots. The height values in square brackets only represent secondary maxima. In some cases the corresponding average contribution function partly exceeds the upper boundary of the model. The missing contribution is in the range of $0.5 \%$ to $2 \%$ for data marked with * and $>2 \%$ for data marked with ${ }^{* *}$.

\begin{tabular}{|c|c|c|c|c|c|c|c|c|c|c|c|c|c|}
\hline & & \multicolumn{4}{|c|}{$z_{\operatorname{maxc}}[\mathrm{km}]$} & \multicolumn{4}{|c|}{$\left\langle z_{\mathrm{cc}}\right\rangle[\mathrm{km}]$} & \multicolumn{4}{|c|}{$\delta\left(z_{\mathrm{cc}}\right)_{\mathrm{rms}}[\mathrm{km}]$} \\
\hline \multirow[t]{2}{*}{ Model } & \multirow[t]{2}{*}{$\mu$} & \multicolumn{4}{|c|}{$\lambda[\mathrm{mm}]$} & \multicolumn{4}{|c|}{$\lambda[\mathrm{mm}]$} & \multicolumn{4}{|c|}{$\lambda[\mathrm{mm}]$} \\
\hline & & 0.3 & 1.0 & 3.0 & 9.0 & 0.3 & 1.0 & 3.0 & 9.0 & 0.3 & 1.0 & 3.0 & 9.0 \\
\hline \multirow[t]{5}{*}{ A } & $\overline{0.2}$ & 581 & 1145 & 1427 & 1612 & 818 & 1076 & 1137 & 1462 & 159 & 231 & 192 & 161 \\
\hline & 0.4 & 528 & 660 & $1263^{*}$ & $1566^{*}$ & 700 & 921 & $1180^{*}$ & $1336^{*}$ & 161 & 216 & $254^{*}$ & $238^{*}$ \\
\hline & 0.6 & 503 & 621 & $1181^{*},[735]^{*}$ & $1455^{* *}$ & 645 & 842 & $1085^{*}$ & $1250^{* *}$ & 156 & 219 & $269^{*}$ & $271^{* *}$ \\
\hline & 0.8 & 489 & 603 & $713^{*},[1173]^{*}$ & $1377^{* *},[867]^{* *}$ & 611 & 795 & $1025^{*}$ & $1193^{* *}$ & 150 & 217 & $274^{*}$ & $288^{* *}$ \\
\hline & 1.0 & 481 & 588 & $695^{*},[1120]^{*}$ & $1348^{* *},[789]^{* *}$ & 588 & 765 & $987^{*}$ & $1158^{* *}$ & 143 & 211 & $273^{*}$ & $297^{* *}$ \\
\hline B & 1.0 & 501 & 620 & $764^{* *},[\sim 1040-1120]^{* *}$ & $936^{* *}$ & 552 & 692 & $821^{* *}$ & $948^{* *}$ & 119 & 154 & $178^{* *}$ & $192^{* *}$ \\
\hline \multirow[t]{3}{*}{$\mathrm{C}$} & & \multicolumn{3}{|c|}{ LTE electron densities: } & & & & & & & & & \\
\hline & 1.0 & $\begin{array}{l}480 \\
\text { NLT }\end{array}$ & $\begin{array}{l}588 \\
\text { electr }\end{array}$ & $\begin{array}{l}720^{*},[\sim 1040-1200]^{*} \\
\text { densities: }\end{array}$ & $780^{* *},[1356]^{* *}$ & 659 & 829 & $990^{*}$ & $1152^{* *}$ & 134 & 197 & $244^{*}$ & $271^{* *}$ \\
\hline & 1.0 & 492 & 732 & 960 & 1176 & 656 & 868 & 1074 & 1255 & 85 & 117 & 137 & 164 \\
\hline
\end{tabular}

contribution function to be rather complicated. The peaks of the individual low-altitude components of those functions for model A (see Fig. 4) move upwards by roughly $100 \mathrm{~km}$ for each increase of factor 3 in wavelength. But additionally the highaltitude contribution, say above $\sim 900 \mathrm{~km}$, grows strongly with wavelength. The mean formation height $\left\langle z_{\mathrm{cc}}\right\rangle$ therefore increases by $\sim 200 \mathrm{~km}$ with each factor 3 in wavelength.

The average contribution functions for an inclined view $(\mu<1.0)$ in model A are shown in Fig. 4, too. At all wavelengths a similar behaviour is obvious: the low-altitude peak (as most clearly seen for short wavelengths at large $\mu$ ) moves higher up and at the same time decreases its relative contribution until the high-altitude range prevails. Although the latter is much broader, in contrast to the relatively sharp low-altitude peak, a maximum can be defined in most cases. The height of this maximum increases with decreasing $\mu$, just as for the lowaltitude contribution. The mean formation height $\left\langle z_{\mathrm{cc}}\right\rangle$ increases by approximately $200 \mathrm{~km}$ with each factor 3 in wavelength for all inclination angles.

The contribution functions for the snapshot from model B, which are shown in Fig. 5, are similar to those for model A, considering that one compares an average over 60 time steps with a single snapshot. Also the formation heights agree generally (see Table 4), although a factor 3 in wavelength only increases the mean formation height by $\Delta\left\langle z_{\mathrm{cc}}\right\rangle \sim 130 \mathrm{~km}\left(\Delta z_{\operatorname{maxc}} \sim 140 \mathrm{~km}\right)$.

Also the contribution functions for the snapshot from model C are similar when using LTE electron densities for the intensity synthesis (see dot-dashed line in Fig. 5). Using the non-equilibrium electron densities from the simulation, however, produces different contribution functions for wavelengths of $1 \mathrm{~mm}$ and longer. At $\lambda=0.3 \mathrm{~mm}$ LTE and non-LTE both are very similar to the other models, reflecting the fact that LTE is still a reasonable assumption for electron densities at that height (LW06). At the longer wavelengths the relevant height ranges of the contributions functions seem to be more localized in the non-LTE case and do not exhibit such a broad range and doublepeaked shape as for the LTE case. The difference is caused by the electron densities which have direct influence on the optical depth. While in LTE the hydrogen ionisation and with it the electron density follow the strong temperature variations of the propagating shock waves, they do vary much less in non-LTE and tend to be at values set by the conditions in the shocks (LW06).
The effect is that surfaces of constant optical depth at wavelengths around $1 \mathrm{~mm}$ are much less corrugated. Consequently, a much smaller height range is sampled in non-LTE whereas in LTE the strongly varying optical depth mixes contributions from low and high layers, depending on the thermal structure along the line of sight (see Fig. 3 by Leenaarts \& Wedemeyer-Böhm 2006b).

The formation heights $z_{\mathrm{cc}}(x, y)$ of all columns in model $\mathrm{C}$ are shown in Fig. 6 in dependence of the resulting brightness temperature $T_{\mathrm{b}}(x, y)$. The strong variation of the electron density and thus the optical depth in the LTE case makes the formation height range increase with brightness temperature. This trend is clearly visible for all wavelengths in the left column of Fig. 6 (LTE). A high brightness temperature is connected to high gas temperature (in a shock front) and thus to an increased opacity. Essentially the atmosphere gets optically thick already high up in the atmosphere if a shock wave is in the line of sight, whereas the cooler temperatures in the post-shock regions allow to look at much deeper layers. In NLTE (right column) this effect is less pronounced for the short wavelengths and essentially absent for the long wavelengths as the fluctuations of the non-equilibrium electron density are smaller. The spread in height is reduced in the NLTE case compared to LTE, e.g. from $\delta\left(z_{\mathrm{cc}}\right)_{\mathrm{rms}}=197 \mathrm{~km}$ to only $117 \mathrm{~km}$ at a wavelength of $1 \mathrm{~mm}$. Still the variation prevents a strict correlation between wavelength and corresponding formation height that would be highly desirable for the derivation of the height-dependent three-dimensional thermal structure of the atmosphere from observations. Nevertheless, the (more realistic) NLTE calculations for model C imply it could be possible at least in a statistical sense. The wavelength dependence of the average formation height remains very similar to the results from model A. An increase by a factor of 3 in wavelength results in an increase of $\Delta\left\langle z_{\mathrm{cc}}\right\rangle \sim 190 \mathrm{~km}\left(\Delta z_{\operatorname{maxc}} \sim 220 \mathrm{~km}\right)$.

The clear tendency of sampling higher layers with increasing wavelength is expected as the opacity goes with the wavelength squared. In principle, the sampled height can be related to the density stratification. An increase of factor 3 in wavelength would then shift optical depth unity by that height after which the density decreased by $3^{2}$. In the models this height is mostly between $220 \mathrm{~km}$ (low chromosphere) and $400 \mathrm{~km}$ (towards high chromosphere) and thus agrees well with the values directly derived from the average contribution functions. 


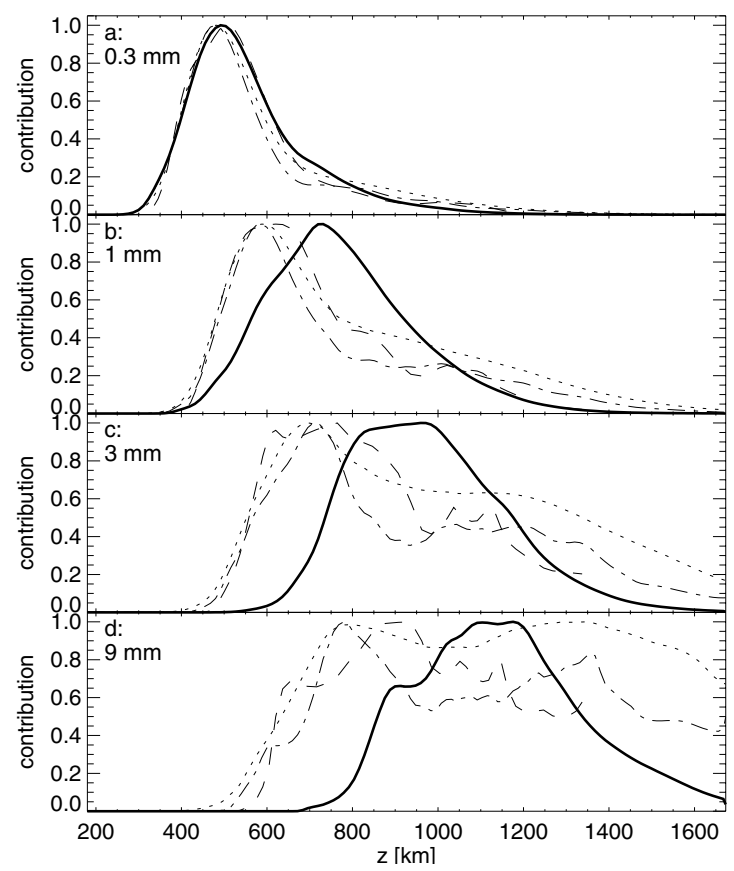

Fig. 5. Normalised contribution functions for continuum intensity on the geometrical height scale for average from model A (see Fig. 4, here dotted line), for the snapshot from MHD model B (dashed), and for the snapshot from the model with non-equilibrium hydrogen ionisation. For the latter the electron densities were first calculated with $\mathrm{RH}$ under the assumption of LTE (dot-dashed) and then with non-equilibrium electron densities available from the model (thick solid). The panels show different wavelengths: a) $0.3 \mathrm{~mm}$, b) $1 \mathrm{~mm}$, c) $3 \mathrm{~mm}$, and d) $9 \mathrm{~mm}$.

\subsection{Temporal evolution}

The analysis of the temporal behaviour relies on model $\mathrm{A}$ as only single snapshots are available for the other models. The dynamics of model $\mathrm{C}$ are the same and also the MHD model B is very similar in this respect.

Pattern evolution time scale. The temporal evolution of the intensity pattern can be quantified in terms of a time in which the autocorrelation decays to the fraction $1 / e$ as it has been done in W04 for gas temperature. Here, we apply the same procedure to time sequences of brightness temperature for all wavelengths and all inclination angles from model $\mathrm{A}$. The time scales are mostly around $23 \mathrm{~s}$ to $24 \mathrm{~s}$ for $\mu>0.2$ and go down to $19 \mathrm{~s}$ for $\mu=0.2$ and $\lambda=3 \mathrm{~mm}$. The extreme case of $17.4 \mathrm{~s}$ for $\mu=0.2$ and $\lambda=9 \mathrm{~mm}$ must regarded as uncertain as it is influenced by possible artefacts due the only partially included formation height range. The gas temperature in the model chromosphere itself exhibits time scales of the same order (20 s to $25 \mathrm{~s})$ and stays almost constant throughout the chromosphere with a tendency towards higher values for the lower layers (see W04). Hence, it is not surprising that the intensity image sequences reproduce roughly the same time scales for the different wavelengths despite the different contribution functions.

Brightness temperature variations. The dynamic nature of the model chromosphere is very obvious if one looks at the temporal variation of the brightness temperature in Fig. 7 (see also Fig. 10 of W04 for the chromospheric gas temperature variation). The amplitude of the fluctuations, expressed quantitatively by means of $T_{\mathrm{b}, \mathrm{rms}}$ (see Table 3, compare with Fig. 9 in W04),
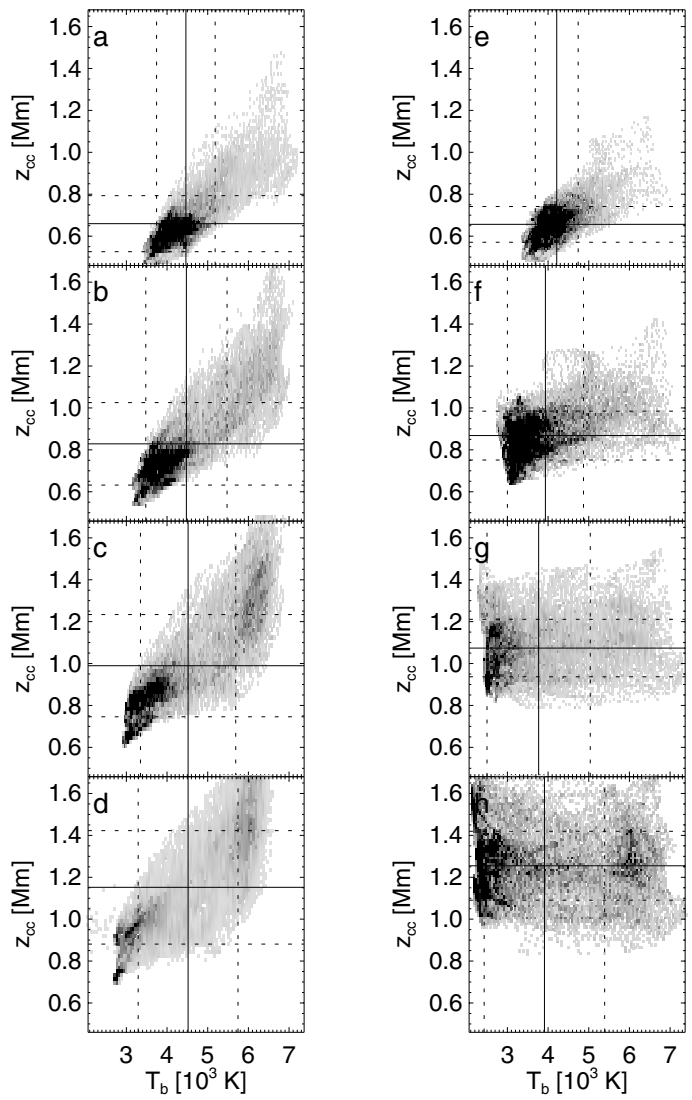

Fig. 6. Formation heights $z_{\mathrm{cc}}$ as function of brightness temperature for the snapshot from model C with LTE electron densities (left column) and non-equilibrium electron densities (right column). The range in brightness temperature and formation height covered by all horizontal positions was divided into bins of $\Delta z=12 \mathrm{~km}$ and $\Delta T_{\mathrm{b}}=50 \mathrm{~K}$. The number of spatial positions with values within the same bin (i.e., the density function) is shown as grey-scale for the wavelengths of $0.3 \mathrm{~mm}(\mathbf{a}, \mathbf{e}), 1 \mathrm{~mm}(\mathbf{b}, \mathbf{f}), 3 \mathrm{~mm}(\mathbf{c}, \mathbf{g})$, and $9 \mathrm{~mm}(\mathbf{d}, \mathbf{h})$. The solid lines represent the average brightness temperatures and formation heights, whereas the dotted lines mark the $1 \sigma$ deviation.

is generally smaller in the lower layers than further up, since the shock waves steepen on their way upwards into the thinner atmospheric layers. The propagating wave fronts are clearly visible as bright streaks in the lower panel which shows the gas temperature in the selected column as a function of height and time. The other temperature enhancements are caused by interaction with neighbouring wave fronts and the fact that the waves do not only move vertically (inside the column) but also laterally and thus can leave and enter the selected column sideways.

Since the continuum intensity at $\lambda=0.3 \mathrm{~mm}$ emerges from the high photosphere and low chromosphere, it exhibits smaller variations of the related brightness temperature compared to the remaining wavelengths which sample higher layers. For the particular example in Fig. 7 we find a standard deviation of $\sim 640 \mathrm{~K}$ at $\lambda=0.3 \mathrm{~mm}$ and around $1000 \mathrm{~K}$ for the longer wavelengths. The vertical dashed lines in the figure mark shock fronts at $\lambda=0.3 \mathrm{~mm}$, which are formed low in the atmosphere (see lower panel). The longer wavelengths show an excess in brightness temperature shortly afterwards. The time difference with respect to $0.3 \mathrm{~mm}$ increases with wavelength and thus with formation height, clearly indicating upward propagating shock waves.

The vertical gas temperature stratification for the selected column is shown in Fig. 8 at a time of $480 \mathrm{~s}$. The shock wave at a height of $z=1000 \mathrm{~km}$ appears as brightening at a 


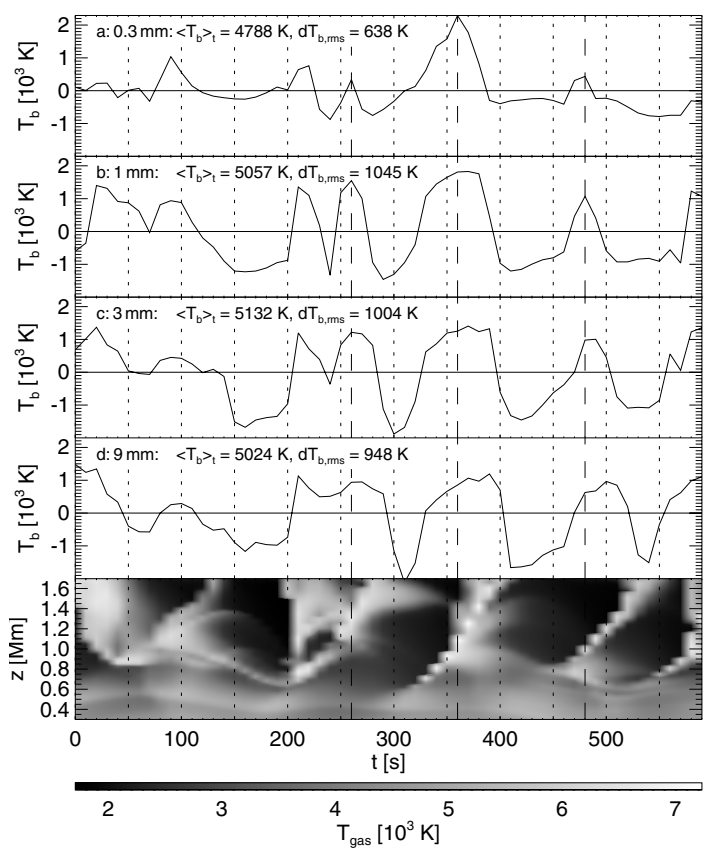

Fig. 7. Temporal variation of excess brightness temperature at a chosen horizontal position ( $x=4460 \mathrm{~km}, y=2020 \mathrm{~km})$ in model A for all four wavelengths (a-d) at disk-centre. In each panel the standard deviation is noted. The lower panel (e) shows the gas temperature (colour legend below) in the selected column as function of time and height. The vertical long-dashed lines mark shock fronts at $\lambda=0.3 \mathrm{~mm}$ and (together with the short-dashed lines at equi-distant times) help to identify the upward propagating fronts after a short delay in the brightness temperature for the longer wavelengths.

wavelength of $1 \mathrm{~mm}$ at $t=480 \mathrm{~s}$ (Fig. 7) and shortly afterwards at the longer wavelengths. The absolute temperature amplitudes of the shock waves, however, depend on details of the numerical modelling of the chromosphere (see discussion in Sect. 6.1). The determination of brightness temperatures from observations will thus provide an important test for the numerical models.

\subsection{Centre-to-limb variation}

In Fig. 9 a sequence of intensity imagesfor $\lambda=1 \mathrm{~mm}$ with increasing inclination angle $\theta$ is shown, illustrating the changes as one goes from disk-centre towards close to the limb. At diskcentre $(\mu=1.0)$ the chromospheric small-scale structure with its bright filaments and dark intermediate regions is best visible, resulting in the highest intensity contrast (see Table 3). But towards the limb the contrast decreases since the dark regions become less dominant. This can be explained if one considers that at disk-centre the chromosphere is seen from above and at (or close to) the limb from the side, making visible different geometrical aspects of the thermal structure of the model chromosphere. From above the model chromosphere appears as alternating pattern of hot and cool gas (see Fig. 8) and thus bright and dark in continuum intensity. There are usually only one or two (or none) shock fronts in the line-of-sight, allowing to look deep into the intermediate dark regions. In contrast from the side (small $\mu$ ) many "propagation channels" of shock waves overlap along the line-of-sight, resulting in much lower intensity contrast (see Fig. 8). Observations of the centre-to-limb variation of the intensity pattern may thus give valuable information on the three-dimensional structure of the solar chromosphere.
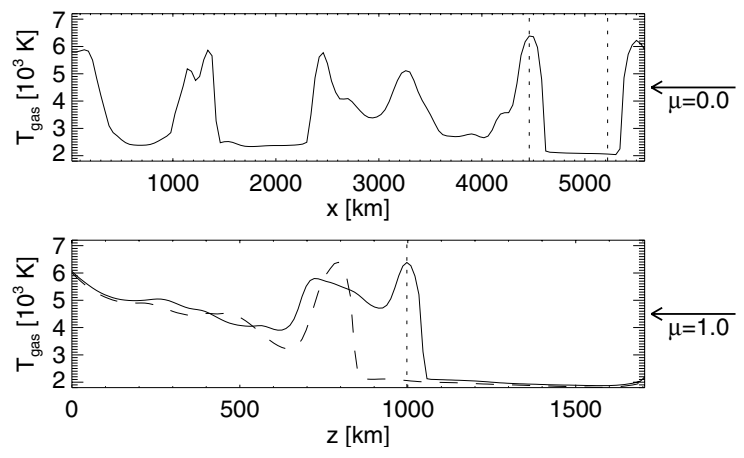

Fig. 8. Gas temperature profiles along the horizontal $x$ axis (upper panel) and the vertical $z$ axis for the same snapshot as in Fig. 1 at $(x, y, z)=(4460 \mathrm{~km}, 2020 \mathrm{~km}, 1000 \mathrm{~km})($ solid) and $(x, y, z)=$ $(5220 \mathrm{~km}, 2020 \mathrm{~km}, 1000 \mathrm{~km})$ (dashed). The positions are marked with dotted lines. The arrows at the right indicate the line of sight direction at the limb $(\mu=0.0)$ and at disk-centre $(\mu=1.0)$.

\subsection{Spatial resolution}

A major disadvantage of past and present instruments for the (sub-) millimetre range is an only poor spatial resolution which hindered a detailed study of the small-scale structure of the solar atmosphere. In order to estimate the spatial resolution which is needed to resolve the pattern clearly present in the model chromospheres used here, we choose a representative intensity image from model A for $\lambda=1 \mathrm{~mm}$ and degrade it artificially by convolution with a Gaussian kernel, mimicking instrumental properties. The result is shown in Fig. 10 for different widths (FWHM) of the Gaussian. The finest structures are already hardly visible at a resolution of $0 \prime$. 3 whereas the larger mesh-like pattern is still discernible at $0 . \prime 9$. The brightness temperature contrast, which is noted next to the panels in Fig. 10, reduces with spatial resolution $\Delta \alpha$. This trend can be fitted with

$\frac{\delta T_{\mathrm{b}, \mathrm{rms}}}{\left\langle T_{\mathrm{b}}\right\rangle} \propto \mathrm{e}^{-\Delta \alpha / D}$

where $D$ is the characteristic length scale of the chromospheric pattern (derived from the fit). This dependence is similar for all wavelengths and inclination angles although the length scale for the best fit is different. Except for $\mu=0.2$, where $D \approx 290 \mathrm{~km}$ for all wavelengths, the length scale increases with wavelength. At disk-centre it increases by $\sim 100 \mathrm{~km}$ for a factor three in wavelength from $730 \mathrm{~km}$ at $\lambda=0.3 \mathrm{~mm}$ to $1030 \mathrm{~km}$ at $\lambda=9 \mathrm{~mm}$. This can be explained by the fact that the propagating shock waves, which produce the pattern, only become visible at the bottom of the chromosphere, whereas in the higher layers the interaction of neighbouring wave fronts developed into a more distinctive pattern of larger mesh-size (see Fig. 2 by W04). On the other hand, $D$ decreases with increasing inclination angle as one looks at the mesh-like pattern from above at disk-centre but sideways on the shock fronts close to the limb (see Sect. 5.4 and Fig. 9).

\section{Discussion}

\subsection{Numerical modelling}

The results of this study demonstrate the constraints on spatial and temporal resolution for observations of the solar inter-network chromosphere with future (sub-)millimetre instruments but should be regarded qualitatively only since some important ingredients are still missing in the models used here. The example of model $\mathrm{B}$ implies that 

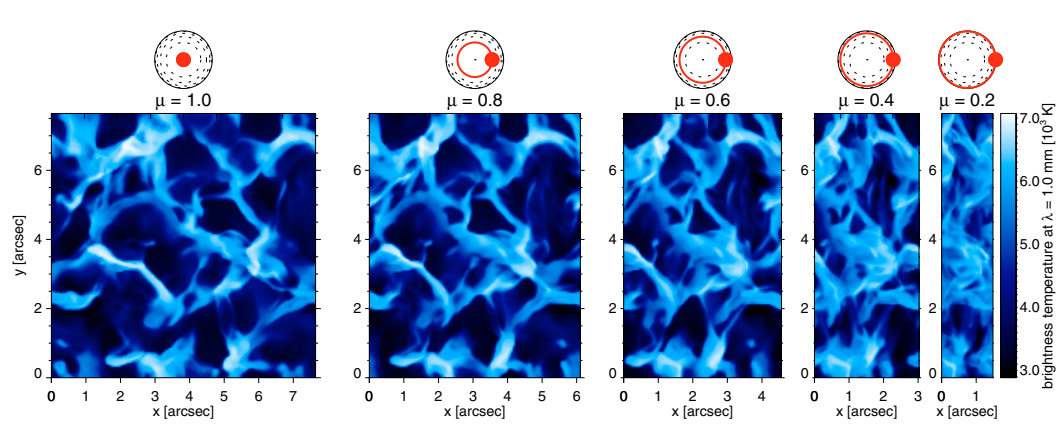

Fig. 9. Variation of continuum intensity maps at a wavelength of $1 \mathrm{~mm}$ with inclination angle $(\mu=\cos \theta)$. The thick circles with solid dot in the sketches above each panel indicate the positions on the solar disk. The different panels sizes are due to foreshortening.

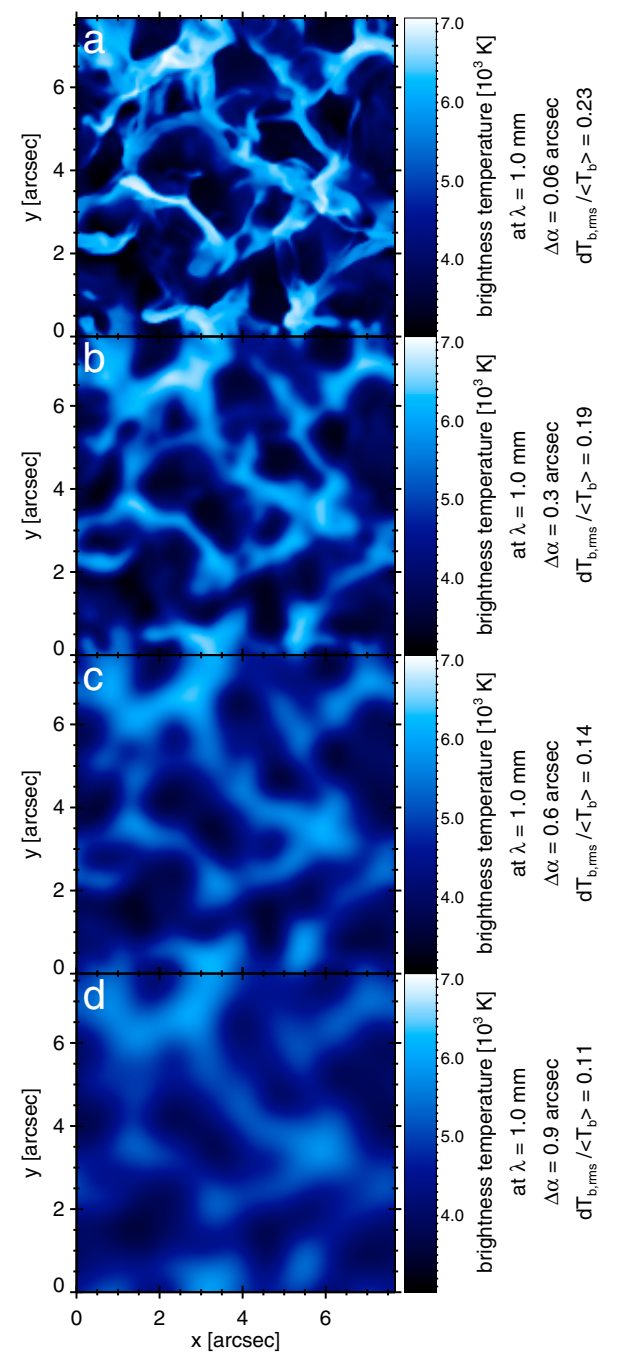

Fig. 10. Intensity image at $\lambda=1 \mathrm{~mm}$ from a single snapshot of model A at different spatial resolutions: a) 0.06 (size of computational grid cells in the original model), b) 0.3 , c) $0 .{ }^{\prime} 6$, d) $0 .{ }^{\prime} 9$. The value range is the same for all panels. The brightness temperature contrast is noted right next to each panel.

even weak magnetic fields, as they are expected for solar internetwork regions, will have most likely important (indirect) influence on structure and dynamics of the chromosphere. Nevertheless, the qualitative picture of pronounced inhomogeneities clearly present in the non-magnetic model A are also found for the weakly magnetic model B (see Sect. 6.2).

A more severe limitation concerns the assumption of LTE for the radiative transfer and the equation of state, which is made here in order to keep the simulations feasible. At chromospheric heights the assumption is no longer valid. Instead a by far more involved treatment of non-equilibrium and radiative non-LTE effects is required (e.g. hydrogen ionisation). This will certainly influence the energy balance and with it the thermal structure of the chromosphere. Taking into account the time-dependence of hydrogen ionisation, which is not instantaneous at chromospheric heights but rather is governed by long recombination time scales, leads to deviations from ionisation equilibrium and thus electron densities that can differ significantly from the corresponding equilibrium values (Carlsson \& Stein 2002; LW06). The effect on the optical depth at millimetre wavelengths is significant, The electron density affects the optical depth and thus determines which layer is effectively mapped. From the analysis of a recent simulations that accounts for non-equilibrium hydrogen ionisation (model C, see LW06) we learn that (i) the height of optical depth unity at a wavelength of $1 \mathrm{~mm}$ is on average close to the LTE case and does not vary that strongly, (ii) the intensity fluctuations and the average brightness temperature are reduced, (iii) the resulting intensity maps are still qualitatively similar (see Sect. 5.1). However, the back-coupling of the nonequilibrium on the equation of state and on the opacities - a final step that has to be done - will most likely increase the peak temperatures of the shock waves.

\subsection{Magnetic field}

The snapshot from magnetohydrodynamic model B gives a first idea about the influence of the weak chromospheric magnetic field when compared to the non-magnetic model A (for $\lambda<$ $9 \mathrm{~mm}$ ). Already the brightness temperature maps in Fig. 1 show a similar pattern for both models. The characteristic length scale of the mesh-like brightenings, which are due to hot shock fronts, seems to be larger in the MHD case. The formation heights are very similar and are only slightly higher in model B although this might be due to the particular snapshot chosen. The average brightness temperature is higher in model $\mathrm{B}$ for $\lambda=0.3 \mathrm{~mm}$ and $1 \mathrm{~mm}$ but is the same than for model $A$ at $3 \mathrm{~mm}$. The average actually decreases with wavelength for model B whereas it increases for model $\mathrm{A}$. The rms brightness temperature variations increase with $\lambda$ in both cases but are $400-500 \mathrm{~K}$ higher in the MHD model. The different behaviour of the two models might be explained with the finding that the magnetic field - although still quite weak - becomes more important with increasing height (S06) and might influence the temperature distribution by suppressing power of the propagating waves. Also the field concentrations in the photosphere might change the wave excitation which should also become visible in the higher layers. The comparison of magnetic and non-magnetic models should be repeated in more detail and with a larger number of snapshots in the future, but alreadya this first qualitative study suggests that high-resolution (sub-)millimeter observations can give 
(indirect) information on the magnetic field topology of the solar chromosphere.

\subsection{Synthetic observations}

The computation of synthetic images that can be directly compared to observations requires more than a simple convolution with a Gaussian (Sect. 5.5), which is strictly speaking only valid in the limit of full $u-v$-coverage (see Sect. 2). For more realistic maps one needs to convolve a synthetic image with the primary beam response of the antenna used, Fourier-transform the result, and multiply with the $u-v$-coverage of the array configuration. The Fourier back-transform yields then the "dirty map". Such a detailed image construction, of course, depends on specific instrumental properties and array configuration at the time of the observation. Nevertheless, synthetic images can be easily generated to match the properties of a particular observation.

\subsection{Comparison to observations and other models}

Loukitcheva et al. (2004) compare the dynamic 1D simulation by $\mathrm{CS}$ and the semi-empirical FAL model A with a large number of observations (see references therein). They conclude that the FAL model A could also account for the measured brightness temperatures, at least when combined with the CS model. Although the brightness temperatures calculated from the FAL A stratification are still within the error bars of the observations, the values tend to be at the upper limit if not even exceeding it. The CS model matches the observations much better as it predicts much lower temperatures than FAL A. The discrepancy is still small for short wavelengths $(\lambda \sim 0.1 \mathrm{~mm})$, where the intensity originates from deep in the atmosphere, but increase to the order of $3000 \mathrm{~K}$ for the longer wavelengths.

The average brightness temperatures in our models is similar to the values for the CS simulation and the observations for the shorter wavelengths $(\lambda \leq 1 \mathrm{~mm})$. For the longer wavelengths, however, CS, FAL A, and also the observed temperatures increase strongly with wavelength whereas our models yield considerably smaller average values of the order $4000-5000 \mathrm{~K}$. In case of $\lambda=9 \mathrm{~mm}$ the discrepancy can partly be attributed to the fact that the continuum forming layers exceed the vertical extent of model A and B and also C in the LTE case although this argument does not hold for the NLTE case for model C. The transition region and its steep temperature gradient, which is included in the CS and FAL A models but not in ours, is a more likely source of discrepancy. In the CS simulation the transition region starts around a height of $1500 \mathrm{~km}$ from where in our models a significant part of the emergent continuum intensity at long wavelengths originates. Consequently the temperatures at long wavelengths would be too low and increase of the average with wavelength not as steep as found by CS and FAL. Last but not least the brightness temperatures distribution is affected by uncertainties in the chromospheric energy balance due to a too simple radiative transfer and the still missing back-coupling of the non-equilibrium hydrogen ionisation to the equation of state. The latter would lead to higher shock peak temperatures.

The brightness temperature variations derived in this work (see Table 3) tend to increase with wavelength (at disk-centre) and thus show the same tendency as found by Loukitcheva et al. (2004). They also explain this behaviour with different formation height ranges increasing with wavelength. For the very long wavelengths $(\lambda>8 \mathrm{~mm})$, however, the temperature variations in the CS model decrease again. The rms variation, shown in Fig. 4 of Loukitcheva et al. (2006) as function of wavelength, actually has a maximum at around $2 \mathrm{~mm}$ with $\delta T_{\mathrm{b}, \mathrm{rms}}=930 \mathrm{~K}$. The corresponding variations in the models presented here are significantly larger for all considered wavelengths. This is related to the differences in the profile and propagation of the shock waves in the 1D model CS and our 3D models. While sawtooth-like shock profiles are clearly present in the CS model (see Fig. 3 by Loukitcheva et al. 2004; and Fig. 1 by Loukitcheva et al. 2006), they are not so easily visible in the models used here (see Fig. 7). In $3 \mathrm{D}$ - in contrast to a $1 \mathrm{D}$ model - shock waves propagate in all spatial directions and interact with neighbouring wave fronts and this way obscure the shock signatures in vertical columns. Nevertheless, there are excess temperatures of more than $1000 \mathrm{~K}$ in both kinds of model. The case of $0.05 \mathrm{~mm}$ for the CS simulation varies less and resembles more a regular oscillatory behavior as it originates from further down in the photosphere.

\subsection{Formation heights}

Double-peaked contribution functions were found by Loukitcheva et al. (2004) for simulated brightness temperature at millimetre wavelengths for single snapshots from the 1D simulation by CS (see Fig. 5 therein). A secondary maximum is also visible in the average contribution for $\lambda=1 \mathrm{~mm}$. This agrees well with the contribution functions for the models used here. Secondary maxima are more obvious in the contribution functions for individual columns, whereas the functions shown in Figs. 4 and 5 are smoother as they represent averages from $3 \mathrm{D}$ calculations with a large number of columns. More than one peak appears if there is more than one shock wave in the line of sight or at least a shock wave above, i.e. at smaller optical depth, the average formation height range set by the background stratification. Multiple contribution peaks are thus a direct imprint of the highly dynamic simulations and details certainly depend on the properties of the shock waves.

A comparison of the average contribution functions for the models by CS and FAL A (Figs. 6 and 7 in Loukitcheva et al. 2004 , resp.) shows that the formation heights are in line with our results with exception of $\lambda=10 \mathrm{~mm}$ in FAL A which is formed at a height of $\sim 2250 \mathrm{~km}$ in the transition region. In contrast we find good agreement for the longest wavelength in our models with the CS model. For the other wavelengths $(0.3-3 \mathrm{~mm})$, however, the average formation height tends to be slightly lower in our models compared to CS and FAL A. According to Loukitcheva et al. (2004), the emission at $\lambda \geq 8 \mathrm{~mm}$ does originate from the transition region which is not included in the models used here. Consequently, a more extended model would be necessary for correctly modelling emission at long wavelengths.

The formation height range for model A seems to be too broad to derive information about the thermal structure from observations. The situation improves when taking into account the non-equilibrium electron density contribution due to hydrogen as this flattens the surface of equal optical depth. As the electrons in chromosphere are predominantly due to hydrogen, non-equilibrium modelling of other electron donators (which are currently treated in LTE) are thus expected to have only a minor influence on the electron number density. But even with an advanced non-equilibrium modelling the contribution functions extend over a significant height range, causing the effective formation heights to vary over $\sim 100 \mathrm{~km}$ (model $\mathrm{C}$, see Table 4). This is illustrated very clearly in Fig. 3 by Leenaarts \& Wedemeyer-Böhm (2006b).

The resulting brightness temperatures should thus be interpreted very carefully as they represent the integrated physical 
state of an extended height range. Hence, a brightness temperature can deviate significantly from gas temperature at a geometric height in the case of an inhomogeneous and rapidly evolving chromosphere.

Nevertheless, our study is promising in the sense that simultaneous observations at many millimeter wavelengths can still serve as a statistical tomography of the chromospheric layers. Not only the wavelength dependence but also the centre-tolimb variation of the formation height might prove useful in this respect.

\subsection{Suggested observations}

Based on the results concerning the spatial resolution and effective formation heights discussed above, we can suggest which will be the most promising instruments (see Sect. 2) for observing the small-scale structure of the solar chromosphere.

Although RAINBOW does access the wavelength range of interest here, the small number of antennae will not allow for the high spatial resolution required for imaging the chromospheric fine-structure. For the same reasons CARMA cannot provide brightness temperature maps that have high spatial and temporal resolution at the same time. EVLA does consist of more antennae, resulting in a much larger number of baselines and thus a much higher spatial resolution. The minimim wavelength of $7 \mathrm{~mm}$, however, is at the limit for our application as a significant part of the continuum radiation at this wavelength and beyond originates from layers that are not or only partly within the computational domain of our models. Nevertheless, EVLA is certainly of interest for observing the upper chromosphere/transition region. In this respect it connects to the wavelength range covered by ALMA. The shortest accessible wavelength of FASR will be even $1 \mathrm{~cm}$. Just as EVLA, it won't be able to map the low and middle chromosphere but will be of great value for investigating the solar corona at even higher spatial resolution than EVLA.

The by far most promising tool for imaging the chromospheric fine-structure at high cadence is ALMA as it can access the spatial and temporal scales implied by our study. The effective spatial resolution, however, might be critical for the longer wavelengths. A possible solution could be the application of Multi-Frequency Synthesis (MFS, see Sect. 2).

The wavelength range of the individual bands of ALMA (see Sect. 2.1) corresponds to an expected difference in formation height range of $\sim 100 \mathrm{~km}$ between lower and upper limit of the band. The thousands of spectral channels by ALMA, which are output simultaneously for each wavelength/frequency band, thus correspond to different layers, stacked with slightly differing formation heights. Tomographic reconstruction methods might then be applied to derive the three-dimensional structure of the layer sampled by the band.

Simultaneous observations in different frequency bands will not be possible but switching between bands can be as fast as $1.5 \mathrm{~s}$ which is fast enough in case of the solar chromosphere. As upwards propagating waves show up in the different bands subsequently with increasing wavelength (see Sect. 5.3), it would be best to cycle through the different bands from high to low frequencies. Tracking waves through the atmosphere should be possible with ALMA. The subsequent appearance of particular features at the different wavelengths will enable the determination of the propagation speed and might - in combination with measured amplitudes - give further insight into wave dissipation and with that into the acoustic heating contribution. Combined simultaneous observation runs with optical diagnostics for the low and middle photosphere have the potential to reveal the excitation sources of waves.

\section{Conclusions}

Based on recent three-dimensional (magneto-)hydrodynamic simulations we conclude that future (sub-)millimetre instruments like ALMA will be capable of linearly mapping the gas temperature distribution in the chromosphere. The temporal and spatial resolution of ALMA will be sufficient to resolve structure and dynamics on scales inherent in the models presented here.

The inhomogeneous and dynamic nature of the (model) chromosphere causes the intensity contribution functions and with that the formation height ranges to be extended and to vary in time and space. The variations, however, stay moderate as the electron density, which mainly defines the optical depth at wavelengths around $1 \mathrm{~mm}$, is connected to the rather well-stratified ionisation degree of hydrogen. The formation height range increases with increasing wavelength. The same effect is found for decreasing inclination angle, i.e. higher layers are sampled close to the solar limb than at disk-centre. This behaviour could be exploited for a (statistical) tomography of the thermal structure of the solar atmosphere. In the case of ALMA the upper photosphere to middle chromosphere will be mapped with the different wavelength bands with high spatial and temporal resolution. The large number of spectral channels within each wavelength band will result in many simultaneous maps which correspond to continuously stacked formation heights. Tomographic reconstruction techniques might then allow volume imaging of the solar atmosphere.

Hence, it should even be possible to track propagating hydrodynamical waves in the solar chromosphere and to study chromospheric oscillations in more detail. This will have import implications for the discussion of the heating mechanism not only for the Sun but for stellar chromospheres in general. The range of application is broad and does include the study of the fine-structure of the magnetic network, too, direct measurement of the magnetic field topology, and even full-disk mosaics. Thus, ALMA will be of great value for finally resolving the discussion about the dynamics and the true structure of the solar chromosphere.

Acknowledgements. In memory of Prof. Dr. H. Holweger. The authors thank (in alphabetical order) T. Ayres, T. Bastian, J. Bruls, M. Carlsson, J. Conway, M. Loukitcheva, R. Osten, O. Steiner, and S. White for helpful comments and discussion. S.W.B. also likes to thank the organisers and participants of the APEX/ALMA meeting in Lund, 2007. S.W.B. was supported by the Research Council of Norway, grant 146467/420, and the Deutsche Forschungsgemeinschaft (DFG), project Ste 615/5.

\section{References}

Al, N., Bendlin, C., \& Kneer, F. 2002, A\&A, 383, 283

Ayres, T. R. 2002, ApJ, 575, 1104

Bastian, T. S. 2002, Astron. Nachr., 323, 271

Beasley, A. J., \& Vogel, S. N. 2003, in Millimeter and Submillimeter Detectors for Astronomy, ed. T. G. Phillips, \& J. Zmuidzinas, Proc. SPIE, 4855, 254 Beasley, A. J., Murowinski, R., \& Tarenghi, M. 2006, in Ground-based and Airborne Telescopes, ed. M. L. Stepp, Proc. SPIE, 6267, 626702

Brown, R. L., Wild, W., \& Cunningham, C. 2004, Advances in Space Research, 34, 555

Carlsson, M., \& Stein, R. F. 1995, ApJ, 440, L29

Carlsson, M., \& Stein, R. F. 2002, ApJ, 572, 626

Conway, J. 2007, priv. comm.

Conway, J. E., Cornwell, T. J., \& Wilkinson, P. N. 1990, MNRAS, 246, 490

Escoffier, R. P., Comoretto, G., Webber, J. C., et al. 2007, A\&A, 462, 801

Fontenla, J. M., Avrett, E. H., \& Loeser, R. 1993, ApJ, 406, 319

Freytag, B., Steffen, M., \& Dorch, B. 2002, Astron. Nachr., 323, 213 
Krijger, J. M., Rutten, R. J., Lites, B. W., et al. 2001, A\&A, 379, 1052

Leenaarts, J., \& Wedemeyer-Böhm, S. 2006a, Proc. of NSO Workshop No. 23, ed. J. Leibacher, H. Uitenbroek, \& R. Stein

Leenaarts, J., \& Wedemeyer-Böhm, S. 2006b, A\&A, 460, 301 (LW06)

Loukitcheva, M., Solanki, S. K., Carlsson, M., \& Stein, R. F. 2004, A\&A, 419, 747

Loukitcheva, M., Solanki, S. K., \& White, S. 2006, A\&A, 456, 713

Mihalas, D. 1978, Stellar atmospheres, 2nd edition (San Francisco: W. H. Freeman and Co.), 650

Rybicki, G. B., \& Lightman, A. P. 2004, Radiative Processes in Astrophysics (New York: John Wiley \& Sons)

Schaffenberger, W., Wedemeyer-Böhm, S., Steiner, O., \& Freytag, B. 2006, in Solar MHD Theory and Observations: A High Spatial Resolution Perspective, ed. J. Leibacher, R. F. Stein, \& H. Uitenbroek, ASP Conf. Ser., 354

Tritschler, A., Schmidt, W., Uitenbroek, H., \& Wedemeyer-Böhm, S. 2007, A\&A, 462, 303
Tsuneta, S. 2006, in 36th COSPAR Scientific Assembly, 3642

Uitenbroek, H. 2000, ApJ, 536, 481

Vecchio, A., Cauzzi, G., Reardon, K. P., Janssen, K., \& Rimmele, T. 2007, A\&A, 461, L1

Wedemeyer, S., Freytag, B., Steffen, M., Ludwig, H.-G., \& Holweger, H. 2004, A\&A, 414, 1121 (W04)

Wedemeyer-Böhm, S., Ludwig, H., Steffen, M., Freytag, B., \& Holweger, H. 2005, ESA SP-560, 1035 (ArXiv Astrophysics e-prints arXiv:astro-ph/0509747)

White, S. M., Loukitcheva, M., \& Solanki, S. K. 2006, A\&A, 456, 697

Wöger, F., Wedemeyer-Böhm, S., Schmidt, W., \& von der Lühe, O. 2006, A\&A, 459, L9

Woody, D. P., Beasley, A. J., Bolatto, A. D., et al. 2004, in Astronomical Structures and Mechanisms Technology, ed. J. Zmuidzinas, W. S. Holland, \& S. Withington, Proc. SPIE, 5498, 30 